\title{
El reto de la formación profesional de los administradores
}

\author{
Mario de Agüero Aguirre*
}

\section{Resumen}

La formación de los administradores en general está orientada, en muchos casos, al desarrollo del conocimiento y habilidades laborales (saber, saber hacer), sin considerar el desarrollo de otras habilidades, así como la promoción de valores y la ética, tendentes a una formación integral que permita una visión global de las condiciones del ámbito económico, político y social dentro del cual se desempeña su profesión.

Palabras clave: administradores, formación, ocupación, conocimientos, habilidades.

Fecha de recepción: 22/01/2007

Fecha de aceptación: 07/01/2008

\section{The Challenge of Professional Managers Education}

\begin{abstract}
Professional managers preparation in general has been focused, in several cases, to the knowledge development and working skils, without considering developing other habilities, as well as ethical values, in order to provide an integrated education to allow a global vision of the economic, political and social conditions framework in performing their professional activity.
\end{abstract}

Key words: managers, management education, professional activity, knowledge, skils.

\section{La universidad y el desarrollo de las profesiones}

Las carreras profesionales no surgieron a partir de los medievos como consecuencia del desarrollo de las universidades; en aquel tiempo (el trivium y el cuadrivium), el enfoque que tenían las universidades sólo atendían a aquellas disciplinas que se consideraban parte de las artes liberales de acuerdo con la tradición romana. Las

\footnotetext{
* Investigador de la División de Investigación de la Facultad de Contaduría y Administración de la Universidad Nacional Autónoma de México. Correo electrónico: mariodeaguero@prodigy.net.mx
} 
profesiones tuvieron su origen en lo que se conocía como los gremios —que después evolucionaron en las artes y los oficios- para desembocar en lo que ahora conocemos como profesiones y que en los países europeos llevó a la creación de las escuelas politécnicas y a la generación de la especialización debido al surgimiento de nuevos conocimientos y al desarrollo de la tecnología (Gómez, 1998).

En esta forma, se estableció una diferencia en la formación disciplinaria, no necesariamente relacionada con el desarrollo económico y el nacimiento de las carreras profesionales, pues fue la realidad socioeconómica la que dio nacimiento a lo que hoy conocemos como profesiones, aunque algunas disciplinas dentro de este contexto se profesionalizaron. Las profesiones están más orientadas a transformar la realidad que a conocerla (con un sentido filosófico) impregnadas de una visión pragmática enfocada al logró de resultados. Se desarrollan partiendo de la experiencia que se va generando en el proceso evolutivo de la sociedad y la economía. Sin embargo, debido a la necesidad de dar a los profesionales una formación más integral en conocimientos, desarrollo de capacidades y de habilidades, así como educación en valores, las universidades fueron incluyendo en sus planes de estudio programas de una variedad de carreras en el nivel licenciatura y de posgrado con la intención de dar una respuesta eficiente a la demanda de sus servicios por parte de las organizaciones tanto del sector público como del privado y de la sociedad en general.

Al irse consolidando el proceso de formación de las universidades se fue desarrollando la ideología, la cual durante varios siglos mantuvo muchas de las características significativas de su pasado clásico. El concepto grecorromano, basado en el cultivo de las artes liberales, fue adoptado por los humanistas cristianos del siglo XvI; especialmente destacan los jesuitas. El trabajo de los humanistas del renacimiento logra la recuperación de gran parte del quehacer clásico. Los avances tecnológicos de los gremios artesanales proporcionan saber, organizados ahora y con mayores posibilidades de difusión (Bowen, 1992: 32-33).

Es, por lo tanto, relevante enfatizar los tres objetivos sustantivos de una universidad: la investigación, la docencia y la difusión del conocimiento. Una institución de educación superior que no incluya a la investigación como una actividad sustantiva no puede ser considerada como tal. La búsqueda del conocimiento, el encuentro con la verdad, es la actividad relevante en una universidad. A través de la vinculación de la investigación y de la docencia se logra la difusión del conocimiento, tanto teórico como práctico. Esa vinculación entre la investigación y la docencia es el fermento en la creación de una conciencia en los estudiantes, de un compromiso para apoyar desde la academia en la solución de problemas de la sociedad. 
El reto de la formación profesional de los administradores

La trascendencia de la universidad como formadora de profesionales que tengan el perfil en función a los requerimientos de la sociedad y del sector empleador - saber, saber hacer, saber ser- demanda que las instituciones de educación superior, de acuerdo con su misión, filosofía y modelo educativo, anticipen y busquen satisfacer los requerimientos del futuro y que esto se traduzca en planes y programas de estudio que, acompañados de la didáctica y el uso de la tecnología de un cuerpo académico con un conocimiento profundo de la disciplina que enseñan y de habilidades docentes, logren que, por medio de la vinculación universidad-empresa, se cubran los requerimientos laborales y sociales de una formación sólida e integral de los administradores.

\section{El aspecto humanista}

Con el desarrollo industrial, el crecimiento de los mercados y la mayor complejidad de las organizaciones, en especial de las empresas, se va desarrollando la función de los administradores, ante la imposibilidad de que los dueños o accionistas de las empresas asuman las funciones cotidianas de la gestión de sus negocios. Esta necesidad hace surgir la profesión de los administradores, lo que da pie al nacimiento, en las universidades, de un abanico de planes y programas de estudio orientados a la función administrativa en sus diferentes fases (v.gr. finanzas, mercadotecnia, recursos humanos, operaciones, comercio internacional). Así se fue consolidando el diseño de planes y programas de estudio en las universidades, dándole una estructura académica a la formación de administradores con un acervo de conocimientos referentes al vasto campo de la economía y del mundo de los negocios.

Como parte de una formación integral se incorporó el aspecto humanista. La formación humanista en las universidades se consolidó y llegó a ser parte de su razón de ser mediante la enseñanza de la filosofía y de las artes en general. Sin embargo, el progreso de las ciencias y de la técnica dio origen a los institutos tecnológicos, en los que ha predominado la enseñanza del conocimiento orientado a la adquisición de conocimientos y habilidades para, fundamentalmente, satisfacer las necesidades del mercado laboral, el cual empezó a demandar profesionales en administración para cubrir los requerimientos de un sector cambiante debido a los avances tecnológicos que se empezaron a introducir en el ámbito de las actividades económicas como consecuencia del desarrollo científico.

En este contexto surge y adquiere forma el objetivo de construir un mundo más humano. La preocupación por combatir las injusticias sociales iba siempre acompañada por el noble objetivo de construir un mundo mejor. Existen antecedentes de que, desde los inicios del siglo ix hasta el siglo XVI, se dio un desarrollo en el aspecto de la formación humanista. Este tipo de enseñanza se dividía en dos grandes campos: el 
trivio basado en la enseñanza de la gramática, la retórica y la dialéctica, y el cuadrivio orientado a la enseñanza de la aritmética, la geometría, a veces mezclada con la geografía, la astronomía y la música (Gómez, 1998). Fue en los siglos XIx y xx cuando a raíz de los efectos, primero, de la Revolución Industrial y, segundo, como consecuencia del gran desarrollo tecnológico y de sus impactos sociales, económicos y culturales, que algunos pensadores y especialistas en el campo de la educación retomaron el tema del humanismo y la educación humanista con el fin de darle al hombre, en su quehacer en la sociedad, un marco más amplio con un contenido que promoviera el desarrollo integral de las personas, cualquiera que fuera su actividad o trabajo.

En un inicio, los programas de formación estaban enfocados fundamentalmente a transmitir los conocimientos relacionados con la gestión de las organizaciones. El campo de acción de los administradores es muy amplio y abarca prácticamente todas las áreas que comprenden el quehacer relacionado con la gestión de las organizaciones, desde la investigación del mercado, los procesos de comercialización, los aspectos relacionados con la administración de la producción y la tecnología, siguiendo con la estructuración de los sistemas de planeación y de información para la toma de decisiones, la administración financiera y la participación en los mercados para la obtención de los recursos monetarios que requiere la empresa y, en forma especial, por su importancia, la formación y desarrollo de capital humano - que es el que mediante el esfuerzo, la visión y la toma de decisiones- genera valor económico, o sea riqueza.

El ingrediente de una formación humanista, integradora y totalizadora - para que en la preparación del administrador se provea de una visión que parta de la dignidad de la persona que recibe los efectos, positivos o negativos de las decisiones y acciones que se toman en el ámbito de los negocios- viene a ser un elemento que permite esperar que se logre un comportamiento en el mundo de los negocios en el que la ética y los valores de solidaridad y subsidiaridad se tengan en consideración en el momento de las decisiones para que éstas tengan un sentido humano y no sólo se tomen en función de la generación de valor económico para unos cuantos, sino que se evalúe el beneficio para la sociedad en general como un todo.

Si partimos de los conceptos anteriores, y nos constituimos en el marco del campo de actuación de los administradores en general, consideramos que la formación en valores es una parte importante de la educación integral de los estudiantes para que se logre transmitir un sentido de búsqueda del bien, de distinguir entre lo correcto y lo incorrecto, de lo justo y lo injusto, entre el bien y el mal. Por lo tanto, y dada la trascendencia en el ejercicio profesional de los administradores en el amplio campo de la actividad económica, es pertinente que sea incluido el manejo de valores y en 
El reto de la formación profesional de los administradores

especial el aspecto ético en los programas de su formación universitaria, lo cual permitirá permear un sentido de búsqueda del bien, pero no sólo individual, sino también del bien común, infundiendo el concepto de compromiso con la sociedad.

Detrás del concepto de una educación integral y humanista, está el cuestionar desde el punto de vista filosófico qué es el hombre para definir cuál debe ser el tipo de educación que le dé un desarrollo en todo su potencial, considerándolo inserto en la sociedad. Consecuentemente, se deberán plantear preguntas claras y algunas hipótesis sobre lo qué es el hombre y lo qué es la vida, lo que sabemos del universo, lo que significa la historia humana y otros aspectos insoslayables para encontrar el sentido de la vida (Meneses, 1979: 173-174).

Las condiciones que presenta el contexto actual —en que se encuentra el campo de acción de los profesionales de la administración- establecen requerimientos que van más allá de una simple visión general de las áreas en que está integrada la actividad de las empresas y las características del entorno. Se requiere darles a los administradores un nivel de solidez y profundidad de competencias, que rebasa el tener un conocimiento general y amplio de las condiciones de los mercados y el proceso de la actividad de la empresa en lo relacionado con la comercialización, producción, finanzas y formación y desarrollo de capital humano. Por lo tanto, es necesario que se apliquen nuevos enfoques al hacer la revisión de los planes de estudio de las licenciaturas, así como de los programas de posgrado.

En el informe que presentó la Comisión Internacional sobre la Educación para el siglo XXI de la UNESCO, titulado Educación: un tesoro escondido, se afirma que se debe:

[...] definir la educación no sólo como se ha hecho hasta ahora, desde el punto de vista de su impacto en el crecimiento económico, sino de la perspectiva más amplia del desarrollo humano. El mundo actual con frecuencia, de manera inconsciente, anhela, a veces sin expresarlo, un ideal y unos valores que podemos denominar 'morales 'Es la noble tarea de la educación, alentar a todos y cada uno a que, actuando conforme a sus tradiciones y convicciones $y$ con cabal respeto al pluralismo, eleven sus mentes y su espíritu al plano de lo universal y, en cierta manera, se trasciendan a sí mismos. La educación tiene otros propósitos que el de proveer mano de obra calificada para la economía; debe servir para convertir a los seres humanos no en el medio para el desarrollo, sino en la razón de ser de éste. La educación es un bien comunitario, un activo que pertenece a todos, que no puede ser regulado sólo por la fuerza del mercado. (1996) 
El concepto subyacente en la educación humanista de los estudiantes es el de formar personas que buscan fundamentalmente desarrollar una actividad que beneficie a los demás, y no solamente buscar logros y satisfacciones personales, sino que se preocupen por los miembros de la sociedad en general. El objetivo de la formación humanista es el de integrar a la sociedad a un profesional moderno, de mente abierta, con iniciativa y que se adapte al cambio. En pocas palabras, un hombre preparado para ser útil a la sociedad, sin menosprecio de desarrollar una actividad realizadora plenamente y que genere ingresos para un nivel de vida satisfactorio. No basta saber, ni saber hacer; se requiere saber ser, con plena conciencia de la dignidad como persona y pleno respeto a la dignidad de los otros. (Meneses, 1979)

En México, se ha venido dando un debate en los últimos años con respecto al enfoque que debe dársele a la formación en las carreras profesionalizantes, debido a la necesidad de mejorar los niveles y la calidad de los egresados de licenciatura en las universidades, a la luz del proceso de globalización e integración económica que se está dando en el ámbito mundial como consecuencia de la formación de uniones y tratados de libre comercio. Lo anterior presiona a un proceso de convergencia de los marcos jurídicos, las prácticas comerciales y los programas académicos de formación de profesionales. Esto ha generado presiones para que las universidades del país redefinan sus objetivos y revisen los currículos académicos para cumplir su misión en relación con las nuevas condiciones de la globalización.

De acuerdo con lo anterior, el diseño de los planes de estudios en el campo de las carreras profesionalizantes en general tiene que partir de un estudio de las condiciones del ámbito con el fin de determinar cuáles son los requerimientos con base en las tendencias que se visualizan en un futuro inmediato. Éste será un elemento para definir el perfil del egresado con los matices y enfoques derivados de la filosofía y modelo educativo de las Instituciones de Educación Superior (IES), lo que ayuda a establecer las competencias que se pretende desarrollar para que se logren satisfacer los requerimientos del entorno laboral, así como las del ámbito social en general.

La necesidad de que exista congruencia entre lo que se enseña en las universidades y la realidad del contexto existente implica que los programas educativos deberán conectar al estudiante con esta realidad social de su ámbito. Al respecto, Dewey opina que:

[...] si un alumno aprende cosas de los libros de texto simplemente en conexión con las lecciones escolares y para repetir lo aprendido cuando se le pida, entonces el conocimiento tendrá efecto sobre alguna conducta, a saber, sobre la de repetir afirmaciones a petición de los demás. No es nada sorprendente que tal "conocimiento" no tenga mucha influencia en la vida fuera de la escuela. 
El reto de la formación profesional de los administradores

[...] En primer lugar, la escuela tiene que ser una comunidad de vida con todo lo que esto implica. Las aspiraciones y los intereses sociales sólo pueden desarrollarse en medios auténticamente sociales, el que se da y al que se toma en la formación de una experiencia común. [...] El aprender en la escuela debería continuarse con el de fuera de ella. Debería haber un libre juego entre los dos. (Dewey, 1995: 270-278)

Diversas instituciones - tanto nacionales como internacionales, atentas a los cambios que con motivo de los avances científicos y tecnológicos se han venido generando- han elaborado estudios acerca de la orientación y enfoque que se le debe dar a la formación de profesionales conectados con la actividad económica (OCDE, 2000: 1-2). Desgraciadamente, no siempre estos estudios consideran la necesidad de una formación integral y el desarrollo de competencias con el fin de que los futuros líderes en el campo de los negocios desarrollen una sólida sensibilidad acerca de la trascendencia de sus decisiones y del amplio marco de sus efectos en la sociedad.

En el caso de las escuelas de administración o de negocios, salvo algunas excepciones notables, no se desarrolla la investigación. Generalmente, a través de su vinculación con el sector productivo de la economía, promueven el diseño de proyectos orientados a mejorar los procesos de gestión de las organizaciones, los procesos de producción, su participación en el mercado, la ingeniería financiera para incrementar la generación de valor económico para el capital financiero; pero poco se ha hecho en el sentido del estudio y formación de teoría de las disciplinas y técnicas relacionadas con la administración en general. Un pragmatismo ha invadido a las universidades que han orientado su actividad al desarrollo de proyectos curriculares de acuerdo con una demanda inmediata del mercado, descuidando no sólo los aspectos relacionados con la investigación, pura y aplicada, para desarrollar nuevas teorías, sino que los programas tanto de pregrado como de posgrado se han orientado a la trasmisión de un conocimiento situado.

En la actualidad, podemos decir que la formación de los administradores está orientada - en la mayoría de los casos - al desarrollo de conocimientos y habilidades laborales (saber, saber hacer) sin darle un peso adecuado al desarrollo de otras competencias, así como a la promoción de los valores y de la ética, tendentes a una formación que permita una visión global de las condiciones del ámbito económico, político y social dentro del cual se desempeñan las empresas. El enfoque, enfatizado en los contenidos como elemento fundamental de la transmisión del conocimiento, puede ser uno de los factores que inciden en la falta de concordancia entre los requerimientos de los empleadores y la formación que reciben los administradores en las Instituciones de Educación Superior (IES). Muchos autores como Mintzberg (2004) se han caracterizado desde hace algunos años por sus críticas a los programas de 
formación de los administradores (MBA) en las universidades estadounidenses, pues en múltiples casos las IES ponen el énfasis en la transmisión de conocimientos muy puntuales sobre los aspectos técnicos y operativos en las empresas sin preocuparse por desarrollar en los estudiantes lo relacionado con habilidades como, por ejemplo, liderazgo, visión global, pensamiento estratégico, trabajo en equipo, aprendizaje permanente, apertura y flexibilidad ante los cambios y, en forma especial, los aspectos relacionados con actitudes y valores (saber ser) para una actuación basada en una ética con sentido social.

La administración, como profesión universitaria, es muy joven y ha evolucionado en forma acelerada, siendo actualmente una profesión reconocida y demandada; pero no ha logrado cabalmente satisfacer los requerimientos de la sociedad y, en especial, de los usuarios de sus servicios, por lo que es necesario hacer un análisis de los nuevos requerimientos dentro del ámbito económico, las características de las organizaciones en el contexto actual y los avances científicos y tecnológicos. Es pertinente, por lo tanto, llevar a cabo un análisis amplio sobre el perfil del administrador mediante la formación de grupos de estudio multidisciplinarios que permitan proponer alternativas orientadas a satisfacer los requerimientos de la sociedad y en especial del sector empleador dentro de un contexto de cambios acelerados en el mundo de la actividad económica, así como en los avances científicos y tecnológicos, especialmente en el campo de la tecnología de la información y la comunicación.

Consecuentemente, las decisiones que se generan en las organizaciones en general -especialmente en las empresas que son tomadas por los administradores- trascienden las fronteras de su actividad económica y producen efectos sociales. Generalmente, no se consideran las implicaciones sociales de estas decisiones porque los criterios que las sustentan están basados en el objetivo de la generación de valor económico. Por lo tanto, pueden dar por resultado un deterioro en el medioambiente (efectos ecológicos), producir un impacto en los niveles de bienestar (estabilidad del empleo), una menor salud (obesidad, efectos colaterales de medicamentos, adicciones en general, etc.). Finalmente, estas decisiones repercuten en una sociedad en la que predomina el individualismo y la no-preocupación por el otro, en un marco de conducta orientada al hedonismo y a la búsqueda de la satisfacción de los deseos individuales. Todo esto tiende hacia una sociedad desarticulada y sin cohesión, soportada por un materialismo egoísta y poco preocupado por las condiciones de bienestar en general y los aspectos de conservación del medioambiente. En las empresas no se puede relativizar el aspecto ético en las decisiones. Detrás de cada decisión está subyacente el aspecto ético, y surgen las preguntas con respecto a sí la decisión es justa o afecta negativamente a otros o hace daño al medioambiente. Debe generarse un valor social que contribuya a crear una sociedad mejor, más libre, más justa y con menores asimetrías en los niveles de bienestar. 
El reto de la formación profesional de los administradores

Los nuevos requerimientos del contexto actual demandan la formación y desarrollo de administradores con un diseño y enfoque que hagan posible que su oferta sea útil no sólo a los usuarios de sus servicios, sino a la sociedad como un todo, por lo que no se debe minimizar el objetivo de buscar darles una formación integral, proporcionándoles un peso adecuado al aspecto ético y al desarrollo de competencias orientadas al liderazgo, trabajo en equipo, actitud abierta al cambio e innovación. La formación de los administradores para asegurar un buen desempeño cuando se incorporan al mercado laboral es una necesidad que tienen que cubrir las instituciones de educación superior. Esto demanda una vinculación con las empresas en general con el fin de que se creen diseños de los programas de estudio adecuados a los requerimientos del mercado laboral, así como también en forma significativa de las necesidades sociales para promover un desempeño de los administradores orientado a la permanencia de las empresas y el aprovechamiento óptimo de los limitados recursos de que disponen. Una mala decisión en la empresa tiene una trascendencia y un costo social.

Una formación sólida e integral de los estudiantes de las disciplinas relacionadas con el vasto campo de la administración - futuros tomadores de decisiones en las empresas - se logra desarrollando, además de los conocimientos inherentes a su profesión, una serie de destrezas, habilidades y actitudes, por lo que se requiere aplicar los métodos didácticos que promuevan un alto grado de congruencia entre el perfil de egreso establecido en el programa de estudios y la metodología didáctica utilizada para el desarrollo de las competencias profesionales.

El grado de eficiencia de un administrador inicialmente descansa en sus conocimientos técnicos; es básicamente un especialista. Pero para su desarrollo y crecimiento en el campo del ejercicio profesional, requiere cada vez más una preparación más amplia, más humanista, más general. Debe darse una educación que englobe todo aquello que dentro de la sociedad contemporánea permita la transmisión del saber y la formación del individuo, una educación prospectiva orientada a las necesidades del futuro.

De acuerdo con lo anterior, el objetivo del desarrollo de ciertas competencias para promover una formación que considere los aspectos relacionados con las actitudes y la cuestión valoral, además de las habilidades necesarias, no se ha logrado totalmente. Resulta por lo tanto pertinente - dadas las condiciones que muestra este contexto- lograr que los efectos que se generan como consecuencia de las decisiones que se toman en las empresas no perjudiquen a los agentes que colaboran en la actividad económica que desarrolla la organización: empleados, clientes, proveedores y el fisco, así como en la sociedad en general. 
Aunque generalmente se pone el énfasis en el campo de los conocimientos específicos de la actividad de los administradores, hay algunos aspectos que no muestran los currículos de los egresados de las licenciaturas relacionadas con la gestión de las organizaciones. Desde hace muchos años se ha venido generando cierta insatisfacción de los empleadores (Mintzberg, 2004), ya sean despachos de asesoría de negocios, empresas comerciales e industriales, intermediarios financieros - derivada de las altas expectativas que se crean de los egresados de las escuelas de negocios-generándose una ambición económica desmedida de los administradores y cierta arrogancia y soberbia al creer que basta el grado académico para ocupar un puesto ejecutivo en los que se tienen que tomar decisiones importantes, trabajar con equipos multidisciplinarios y, en el contexto actual, con grupos policulturales.

En el campo de la formación de los administradores es común encontrar que las IES ofrecen planes y programas de estudio orientados a formar profesionales que satisfagan los requerimientos de un mercado laboral. Con este enfoque ocurre que se concentran en la transmisión de conocimientos que parten de los contenidos establecidos en los mapas curriculares o en los silabus o cartas descriptivas de cada asignatura que integra el programa. Aunque en muchos casos hablan de un perfil de egreso del estudiante con el desarrollo de competencias, valores y actitudes, en muchas ocasiones -en los planes de estudio y en la metodología didáctica- no se encuentran elementos para evaluar cómo se desarrollan esas otras competencias. Lo mismo ocurre en relación con los valores y la ética, pues aunque es común encontrar en los perfiles de egreso conceptos relacionados con el desarrollo de valores y ética, no existen en la actividad docente los medios y la didáctica para transmitirlos. Habría que establecer una congruencia entre el perfil que se pretende desarrollar en el estudiante y el requerido para lograr el objetivo de desarrollar habilidades, valores y actitudes.

En la formación de los administradores, dado el carácter profesionalizante de su formación y su enfoque pragmático, reviste especial interés analizar las tendencias educativas apoyadas en las tecnologías de la información y la comunicación (uso del software, simuladores, Internet), considerando que además en el ámbito de las organizaciones en general está ocurriendo una verdadera revolución tecnológica que requiere ser tomada en cuenta en la elaboración de los planes y programas de estudios de estos profesionales. Esto se vuelve más relevante dada la velocidad con que ocurren estos cambios y la insatisfacción manifiesta, principalmente en el sector empleador, sobre la solidez de la preparación que reciben los estudiantes en su formación en las IES. Esta insatisfacción ha sido recogida y expresada por diversos autores, como Mintzberg (2004) que se han adentrado en los aspectos de la formación de los administradores, tanto a nivel de pregrado como al de posgrado. 
El reto de la formación profesional de los administradores

\section{Los nuevos requerimientos}

Desde hace ya algún tiempo tanto en el ámbito académico como en el mundo empresarial nacional e internacional, sobre todo en los Estados Unidos de América, se viene discutiendo si los administradores egresados de las IES cubren los requerimientos actuales de los negocios. El mundo empresarial es muy dinámico; los cambios constantes que se vienen generando por la globalización de los mercados y la aparición de nuevas tecnologías van demandando de los administradores competencias diferentes y van requiriendo, cada vez más, el desarrollo de éstas hacia los aspectos de liderazgo, negociación y una visión más amplia en cuanto a las diversidades culturales, costumbres, prácticas y manejo de otras lenguas. Por esto, cuando se habla de educación universitaria se está hablando de una formación integral de la persona, proporcionándole un desarrollo armónico entre los aspectos intelectuales, espirituales, psicológicos y físicos para dar a este capital humano - producto final de las universidades - las opciones para una realización personal y no sólo capacitar para un mercado de trabajo cada vez más competitivo y demandante. Se requieren personas educadas y profesionales eficientes para garantizar una buena administración de las organizaciones y la maximización de los beneficios para los diferentes agentes que participan en la actividad económica: los inversionistas, los proveedores, los consumidores, los trabajadores, entre otros.

Milton Friedman menciona en un artículo que apareció en la revista Fortune que:

[...] los malos sistemas educacionales hacen que nuestro promedio de ingreso sea inferior al que podría ser. [...] por lo que será fundamental tener un sistema educativo eficiente y moderno, a fin de que los individuos en todo el país tengan las mayores oportunidades de usar sus propios talentos y habilidades. (Friedman, 2001)

Por su parte, Fernando Savater en El valor de educar (1999:145-146) afirma que:

[...] el proceso de enseñanza nunca es una mera transmisión de conocimientos objetivos o de destrezas prácticas, sino que se acompaña de un ideal de vida y un proyecto de sociedad, [...] La educación tiene como objetivo completar la humanidad del neófito; pero esa humanidad no puede realizarse en abstracto ni de modo totalmente genérico, ni tampoco consiste en el cultivo de un germen idiosincrásico latente en cada individuo, sino que trata más bien de acuñar una precisa orientación social: la que cada comunidad considera preferible.” (1999: 145-146) 
En un artículo publicado por la revista Fortune en marzo de 1986, Curtis W. Tarr, en ese entonces decano de la Johnson Graduate School of Management de la Universidad de Cornell, menciona la necesidad de humanizar a los administradores que se forman en las escuelas de negocios de las universidades norteamericanas. Además, señala:

[...] algo ha pasado. Esos jóvenes parecen intentar destruirse unos a otros con tal de llegar a la cumbre. [...] Con frecuencia los jóvenes graduados son muy agresivos y oportunistas. [...] Sin embargo, la mayoría de los responsables en las escuelas de negocios afirman que ellos creen que están haciendo un buen trabajo en la preparación de los estudiantes. Cerca del 80 por ciento dice que debería ponerse un mayor énfasis en la enseñanza de valores humanos y aplicarlos en el campo laboral. (Tarr, 1986: 111-112)

Más adelante, Tarr (1986:112) agrega que el presidente de una gran corporación del sector de transportes piensa que "muchos administradores (maestros en Administración de Negocios: MBA por sus siglas en inglés) parecen creer que buenas habilidades analíticas acompañadas de conocimientos en computadoras son suficientes. Pero definitivamente no lo son". Al final del artículo, el autor concluye que "el administrador tiene que adquirir una preparación más humana para su vida en los negocios [...] Las escuelas de administración deben tener una clara actitud de cambio si quieren fortalecer y humanizar su programa curricular".

Comentarios como los expresados por el autor de ese artículo, hace ya 20 años, se leen con frecuencia en las revistas de negocios norteamericanas y europeas, lo cual es un indicio del grado de insatisfacción en la formación humana de los administradores. México no es una excepción en este aspecto, pues se percibe una gran paradoja entre la filosofía de muchas universidades en cuanto a su objetivo de formación integral y humana de los estudiantes de administración y la preocupación por desarrollar competencias para el mercado laboral, sin darles los elementos indispensables para su formación humanista, dada la naturaleza de la actividad que desarrollan los administradores en el mundo de los negocios.

En el número correspondiente al mes de agosto de 2003 de la Harvard Business Review aparece una serie de entrevistas a directivos de empresas globales líderes, sobre las características que debe tener un ejecutivo en el contexto actual. Llama la atención los comentarios de Stephen Green - Group CEO de HSBC, un importante conglomerado financiero- quien dice que ellos no le ponen mucha atención sobre qué o dónde estudió la gente, sino que lo fundamental en la contratación de sus ejecutivos es la fuerza y el vigor, la iniciativa, la sensibilidad cultural y su visión del mundo desde una perspectiva global (Green, 2003). 
El reto de la formación profesional de los administradores

En el caso de las escuelas de administración o de negocios, salvo algunas excepciones notables, no se desarrolla la investigación. Generalmente, a través de su vinculación con el sector productivo de la economía, promueven el diseño de proyectos orientados a mejorar los procesos de gestión de las organizaciones, su participación en el mercado, la ingeniería financiera para incrementar la generación de valor económico para el capital financiero.

En un artículo aparecido en la Harvard Business Review, sus autores Warren G. Bennis y James O'Toole sostienen que la orientación "cientificista", demasiado centrada en la investigación, induce a las escuelas de negocios a la "contratación de profesores con una experiencia limitada del mundo real, y gradúan a estudiantes que están mal preparados para lidiar con asuntos complejos y no cuantificables: en otras palabras, para lidiar con los verdaderos desafíos de la gestión.” (Bennís, 2005)

Posteriormente, estos autores mencionan que:

[...] hoy los programas de MBA enfrentan una fuerte crítica por fallar a la hora de impartir habilidades útiles, preparar líderes, inculcar normas de comportamiento ético e, incluso, por no lograr buenos empleos corporativos para sus graduados. Estas críticas no sólo provienen de los estudiantes, empleadores y los medios de comunicación, sino también de los decanos de algunas de las escuelas de negocios más prestigiosas de Estados Unidos. (Bennís, 2005)

Esto se debe al énfasis que se pone en los aspectos de contenidos (conocimientos) en el desarrollo de planes y programas de estudio de las disciplinas relacionadas con la administración, sin tomar en consideración el desarrollo de otras competencias porque en el mundo real de la gestión no bastan los conocimientos, se requiere disponer de otras habilidades, como una visión y pensamiento estratégicos y capacidad para trabajar en equipo con grupos multidisciplinarios y policulturales, entre otras cosas.

En el desarrollo de los planes y programas de estudio en el campo de la administración deben tomarse en consideración los requerimientos de un contexto de grandes cambios y generación de nuevas tecnologías, sobre todo en el campo de la información y de la comunicación, cuyos avances han sido aprovechados en el desarrollo de lo que se denomina tecnología educativa, generándose una gran variedad de software que ha permitido hacer más eficiente el proceso de aprendizaje y diseñar programas de difusión del conocimiento, convirtiéndose en una útil herramienta para el aprendizaje mediante sistemas electrónicos en línea o a distancia, que funcionan tanto en forma sincrónica como asincrónica, lo que permite al usuario de lo que se ha dado en llamar "servicio educativo" tener acceso a una variedad de programas, cursos y seminarios sin tener que asistir a una aula ni estar restringido por un horario. 
Sin embargo, uno de los cuestionamientos que se le hace a los sistemas de educación en línea o a distancia, lo que se ha denominado "universidad virtual", es que el papel que se le asigna al profesor es el de un facilitador, relegando su tradicional función de formador; su presencia es esporádica y muchas veces sólo a través de medios electrónicos. Asimismo, se considera que cuando el programa descansa fundamentalmente en el uso de los medios electrónicos, el énfasis se pone en la transmisión de conocimiento, o sea, se le enseña al estudiante a adquirir conocimientos (saber) y aplicarlos (saber hacer), eliminando el aspecto formador (saber ser).

Para hacer un nuevo diseño de los planes y programas de estudio para la formación de administradores, como en cualquier otro caso similar, se tiene que partir de un análisis de las condiciones del ámbito con el fin de determinar cuáles son los requerimientos en el contexto actual y las tendencias que se visualizan en un futuro para poder hacer un diagnóstico. Éste será la base para definir el perfil de egreso de los administradores, con los matices y enfoques derivados de la filosofía y modelo educativo de las IES, que permitirá establecer claramente las competencias que se pretende desarrollar (conocimientos, habilidades, destrezas), así como actitudes y valores para que se logren satisfacer los requerimientos del entorno profesional, así como las del ámbito social en general.

Esto necesariamente obliga a una revisión de los métodos de enseñanza. Las escuelas y facultades de enseñanza de las disciplinas relacionadas con el ámbito de los negocios están enfrentando fuertes presiones para que se modernicen en las técnicas didácticas y atiendan en forma más eficiente los requerimientos de una economía cada vez más especializada, más compleja y cambiante, adecuando el acervo de conocimientos de los administradores del futuro a las nuevas condiciones. No sólo el contenido curricular de los programas está siendo revisado exhaustivamente, sino que se encuentra definiendo un nuevo perfil de los profesores con el uso de la tecnología informática.

Si se parte del perfil que se pretende dar a los administradores que egresen de las IES, se podrá elaborar el mapa curricular donde quedarán establecidas las áreas de conocimiento y, en forma especial, definir cuál es la columna vertebral o área de concentración para trabajar en los contenidos y establecer el peso que las disciplinas que integran el mapa curricular tendrán en el programa de estudio. De esta forma, se construirán los contenidos, elaborando las guías o silabus correspondientes, mediante la definición de las competencias que cada asignatura proporcionará al estudiante determinando qué método didáctico será empleado y cuál es la tecnología o medios que deberán emplearse con el propósito de eficientar el proceso de enseñanza y aprendizaje. 
El reto de la formación profesional de los administradores

Pero para que estos planes y programas de estudio se conviertan en un elemento útil a los estudiantes, es necesario que se cuente con un cuerpo docente preparado didácticamente y con un conocimiento sólido y actualizado de la disciplina que enseñan, así como que en ese proceso de enseñanza se utilicen los medios que aseguren y eficienten el proceso de aprendizaje, apoyándose en la tecnología disponible y aplicable a la asignatura correspondiente (medios visuales o audiovisuales) mediante la práctica de ejercicios, el uso del método del caso, juegos de negocios, simulaciones con el uso computadoras y, en general, la utilización de software desarrollado para apoyar el proceso de aprendizaje.

Debido a lo anterior, se está generando un cambio en el perfil y papel del profesor en los modelos actuales, lo que implica que, además de poseer los conocimientos especializados de la disciplina que enseña, deberá contar con las habilidades didácticas apoyadas en el uso de la tecnología de la información en un proceso enfocado a que el estudiante "aprenda a aprender"; además, orientado a la transmisión de conocimientos conjuntamente con el desarrollo de competencias profesionales (liderazgo, visión, pensamiento estratégico, trabajo en equipo), promoviendo el desarrollo de una visión ontológica que contribuya a desarrollar el saber ser (actitudes y valores), enfatizando el aspecto ético.

A su vez, dados los requerimientos actuales para la formación y especialización de los administradores, es necesario aceptar que - como en el caso de la construcción de un edificio- se tienen que crear unos cimientos sólidos que soporten una estructura de varios pisos. El nivel de pregrado equivale a esos cimientos de la construcción de un profesional, que proporciona un sustento amplio, general y a un nivel de profundidad adecuado para tener una visión completa y clara de las funciones y responsabilidades de los administradores. Posteriormente, combinándose con la experiencia laboral y con base en programas de posgrado, se proporcionará una especialización en los vastos campos de la administración. Por lo tanto, se considera pertinente que una vez obtenido un conocimiento sólido de la actividad en el mundo de las organizaciones, a través de una licenciatura, se ofrezca un abanico de alternativas de programas de posgrado orientados a la especialización.

A la luz del proceso de globalización e integración económica que se está dando en el ámbito mundial, se ha desatado una importante polémica en relación con el enfoque que debe dársele a la educación superior - especialmente en lo referente a la formación de los administradores- como consecuencia de las necesidades de mejorar los niveles de calidad de los egresados de las facultades de administración. Esto ha generado presiones para que las universidades del país redefinan sus objetivos y revisen los currículos académicos para cumplir su misión con base en las nuevas condiciones. 
La importancia en el desarrollo de competencias en la formación profesional permitió el surgimiento de un programa en este sentido en la Unión Europea, que se ha denominado Proyecto Tuning Europa, integrado por 135 universidades que desde el año 2001 llevan adelante un intenso trabajo en pos de la creación del Espacio Europeo de Educación Superior, el cual ha tenido un fuerte impacto al hacer una propuesta sobre las competencias que deben desarrollarse en la formación profesional que se imparte en la educación superior. Dadas su impacto e importancia, surgió el Proyecto AlfaTuning-América Latina integrándose inicialmente a partir de 2003, el cual es una idea intercontinental nutriéndose de los aportes académicos tanto europeos como latinoamericanos para contribuir con el desarrollo de la definición de las competencias genéricas y específicas de cada área de estudio integrada inicialmente en doce áreas temáticas: administración de empresas, arquitectura, derecho, educación, enfermería, física, geología, historia, ingeniería civil, matemáticas, medicina y química (Comisión Europea, 2006).

El desarrollo de competencias se utiliza para alcanzar una formación académica que permita un equilibrio armónico entre conocimientos, habilidades y actitudes (saber, saber hacer y saber ser). En el caso de los administradores, esto se vuelve más relevante porque para lograr un desempeño pertinente, no se depende exclusivamente de los conocimientos, sino de la capacidad para desarrollar otras competencias. Por lo tanto, se manifiesta la necesidad de una formación integral basada en un método de aprendizaje en el que, conjuntamente con la adquisición de conocimientos, se apliquen métodos didácticos apoyados en la tecnología educativa para que el egresado desarrolle un conjunto de competencias encaminado a un desempeño pertinente. El debate con respecto al desarrollo de competencias en los estudiantes de las disciplinas administrativas se centra fundamentalmente en los medios e instrumentos idóneos para lograr que los estudiantes desarrollen las habilidades y actitudes requeridas.

El tema del desarrollo de competencias profesionales es de una relativa novedad en nuestro país. Mucha información sobre el tema se ha desarrollado por autores europeos y norteamericanos como un nuevo enfoque en el proceso de aprendizaje. En el caso de los administradores en general, se ha detectado la importancia de desarrollar —además de los conocimientos- otras habilidades para un desempeño pertinente de los egresados. Las instituciones de educación superior han detectado estos requerimientos y, en general, al definir el perfil del egresado en sus programas de licenciatura destacan estas características. Sin embargo, no aclara si en el proceso de aprendizaje va implícito el uso de una metodología didáctica orientada al desarrollo de competencias. 
El reto de la formación profesional de los administradores

\section{Una formación integral}

La misión y el compromiso de la universidad se centran en lograr una formación integral de los estudiantes de administración para que al insertarse al campo profesional tengan un desempeño pertinente y no sólo se preocupen - aunque no se debe descuidar este aspecto- por la generación de valor económico en beneficio del capital financiero. Se debe buscar el desarrollo de un conjunto de competencias profesionales, así como el fomento de valores y un manejo ético orientados a generar riqueza, pero no sólo riqueza económica, sino también riqueza humana (Cannac,1984).

Desde hace ya algunos años, se han producido ensayos y se han llevado a cabo foros para discutir la problemática de la formación universitaria de profesionales. Al respecto, en uno de esos ensayos se dice que:

Puede afirmarse que hay falta de correspondencia entre el mundo educativo y el mundo del empleo. Dada la heterogeneidad de los procesos productivos de nuestra economía, es irrelevante gran parte de la educación que pretende preparar para ellos. Por el contrario, se observa una creciente devaluación de la educación formal. [...] Por otra parte, tampoco parece haber una correlación directa entre escolaridad y productividad. La diversificación del sistema educativo para producir mandos medios (técnicos) y superiores (profesionales universitarios), tal como se dio, tiende a convertirse en una falacia. En virtud del rápido cambio tecnológico, hay una escasa relación entre la educación y la fuerza laboral y un permanente desencuentro entre los cuadros calificados que forma la escuela y la estructura del empleo. (Aguilar, 1992: 23-24)

En otros ensayos, que forman parte de la compilación realizada por Gilberto Guevara Niebla, se afirma:

Las universidades y centros de educación del país han reproducido un modelo de organización académica de base disciplinaria, que favorece la desagregación del conocimiento y separa la docencia de la investigación. Este modelo ha mostrado una enorme resistencia a la innovación y ha dado lugar a la compartimentación político-académica de los centros de estudio. (Muñoz, 1992:59)

La educación superior para la formación de profesionales, así como las universidades que la proporcionan, han estado sujetas a un cuestionamiento con respecto a la pertinencia de los programas y a la eficacia con que éstos se desarrollan. La estructura curricular en cualesquiera de los casos deberá partir de las necesidades que surgen de los servicios que estos profesionales prestan a la sociedad, considerando que por su naturaleza deberán atender los requerimientos de un entorno en el cual la demanda genera presiones para que - además de que en los currículos se considere el aspecto 
de una formación universal y humanista - se profundice en el estudio de las disciplinas que forman parte del programa curricular específico de cada profesión y se considere la conveniencia de incluir en los planes las prácticas de campo de los estudiantes y el manejo de ejercicios y casos dentro del aula y los laboratorios, utilizando la tecnología, con el objetivo fundamental de introducir al estudiante al mundo práctico. En este aspecto es pertinente, en un proceso de vinculación de la universidad con las empresas, la celebración de convenios para que los estudiantes puedan participar en programas de tiempo parcial en las funciones que componen el quehacer cotidiano de las empresas.

Diversas instituciones, tanto nacionales como internacionales, atentas a los cambios que con motivo de los avances científicos y tecnológicos se han venido generando, han elaborado estudios acerca de la orientación y enfoque que se le debe dar a la formación de profesionistas conectados con la actividad económica-administrativa de las organizaciones. Desgraciadamente no siempre estos estudios consideran la necesidad de una formación integral y el desarrollo de competencias, así como la formación en valores con el fin de que los futuros líderes en el campo administrativo y de negocios desarrollen una sensibilidad acerca de la trascendencia de sus decisiones y del amplio marco de sus efectos en la sociedad. Esto es más relevante a la luz del proceso de globalización económica que se ha estado dando en las últimas décadas, lo cual tiene implicaciones sociales, culturales y políticas.

Al tenor de este nuevo enfoque, la Organización para la Cooperación y el Desarrollo Económico (OCDE) ha realizado un estudio sobre los requerimientos educativos en función de los cambios que están ocurriendo y en el cual plantean cuáles deben ser los elementos que deben contener los currículos académicos en la formación de los profesionales que concurrirán a ese mercado globalizado. En la nueva cultura del manejo del conocimiento — basada en los paradigmas de la globalización y en la tecnología de la información- la OCDE elaboró un estudio referente al manejo del conocimiento en una sociedad del aprendizaje (OCDE, 2000).

En este estudio se enfatiza que "es imperativo un cambio de paradigma educativo, un nuevo enfoque racional, pragmático y articulado a la revolución informática, para rediseñar la educación" (OCDE, 2000: 36). Ante los retos de un mundo globalizado, en el cual se busca una eficiencia económica como requerimiento del mercado, se tiene que buscar un concepto de educación orientado a una sociedad más libre, más democrática y con un sentido ético de la justicia social con el fin de corregir la enorme distancia entre los niveles de bienestar de los que tienen mucho y los que carecen de todo. 
El reto de la formación profesional de los administradores

Por otro lado, la UNESCO, con anterioridad a la OCDE, publicó un estudio que denominó La educación: Un tesoro escondido, también conocido como el Reporte Delors, cuyo nombre se debe a que Jacques Delors encabezó el equipo encargado de la investigación. En la parte introductoria, al referirse a la misión de las IES, se señala:

Al respecto, las universidades ofrecen determinadas peculiaridades que les confieren un carácter excepcional: son el conservatorio vivo del patrimonio de la humanidad, patrimonio que se renueva incesantemente por el uso que de él hacen los profesores y los investigadores. Por lo general las universidades son multidisciplinarias, gracias a lo cual cada individuo puede superar los límites de su entorno cultural original. Asimismo, tienen en general más contacto con el mundo internacional que las demás estructuras educativas.” (UNESCO, 1996: 17)

En ese texto se establece que:

[...] en una sociedad, la enseñanza superior es, a la vez, uno de los motores del desarrollo económico y uno de los polos de la educación a lo largo de la vida. Es a un tiempo depositaria y creadora de conocimientos; además, es el principal instrumento de transmisión de la experiencia cultural y científica acumulada por la humanidad. En un mundo en el que los recursos cognoscitivos tendrán cada día más importancia que los recursos materiales como factores del desarrollo, aumentará forzosamente la importancia de la enseñanza superior y de las instituciones dedicadas a ella. Además, a causa de la innovación y del progreso tecnológico, las economías exigirán cada vez más competencias profesionales que requieran de un nivel elevado de estudios." (UNESCO 1996:144)

Más adelante, en el Reporte Delors, que concuerda con las aseveraciones establecidas en los ensayos compilados por Guevara Niebla (1992), se agrega:

La enseñanza superior está en crisis desde hace una decena de años en gran parte del mundo en desarrollo. [...] El desempleo de los titulados y el éxodo de competencias han acabado con la confianza que se depositaba en la enseñanza superior. El desmesurado atractivo que ejercen las ciencias sociales ha producido desequilibrios entre la categoría de titulados disponibles y de los empleadores, por la calidad del saber que imparten los establecimientos de enseñanza superior. 
A continuación, se menciona que:

[...] las universidades no monopolizan la enseñanza superior, y de hecho, los sistemas nacionales de enseñanza superior son en la actualidad tan variados y complejos, por lo que se refiere a sus estructuras, programas, alumnado y financiamiento, que es difícil clasificarlos en categoría claramente diferenciadas. (UNESCO, 1996: 145)

Por otra parte, cuando se aborda el tema de la tecnología en el proceso de enseñanzaaprendizaje, se enfatiza la importancia de ésta, tratándose de un instrumento valioso para lograr una mayor eficiencia en la obtención de conocimiento. De igual manera, se afirma:

Cada universidad debería volverse abierta y dar la posibilidad de aprender a distancia, en el espacio y en distintos momentos de la vida. La experiencia de la enseñanza a distancia ha demostrado que, en la enseñanza superior, una dosificación juiciosa de medios de comunicación, cursos por correspondencia, tecnología de comunicación computarizada y contactos personales puedan ensanchar las posibilidades ofrecidas por un costo relativamente reducido. Esas posibilidades deben comprender a la vez la formación profesional y una enseñanza que enriquezca mental y espiritualmente a los alumnos. (UNESCO, 1996: 147)

Los autores de este estudio se refieren al papel fundamental que desempeña el cuerpo docente en la educación superior:

[...] es frecuente que en la enseñanza profesional falten profesores calificados en estos campos, lo que contribuye a realzar su prestigio. La formación impartida al personal docente tiende a ser una formación separada que lo aísla de las demás profesiones. Hay que corregir esta situación. Los profesores deberían tener también la posibilidad de ejercer otras profesiones fuera del marco escolar, para familiarizarse con otros aspectos del mundo del trabajo, como la vida de las empresas, que a menudo conocemos mal. (UNESCO, 1996:165)

Tanto en el ámbito de los organismos internacionales, como es el caso de la UNESCO y de la OCDE, así como por parte de las autoridades educativas en el nivel nacional y del conjunto de IES, se ha manifestado una preocupación por la necesidad de aplicar nuevos conceptos en el proceso de enseñanza y aprendizaje. En el caso específico de la formación de administradores, los cambios que se han generado — tanto en la ciencia como en la tecnología y el desarrollo y globalización de la economía - están teniendo un impacto en la forma de manejar las organizaciones en general y en espe- 
El reto de la formación profesional de los administradores

cial las empresas, por lo que se están evaluando los nuevos requerimientos mediante la búsqueda de alternativas que permitan diseñar planes y programas de estudio, tanto de licenciatura como de posgrado con el fin de que los egresados de las escuelas de administración o de negocios tengan el perfil adecuado para un funcionamiento eficiente como participantes en la gestión de las empresas.

En el documento de la OCDE se hacen interesantes consideraciones sobre el papel de las IES en esta sociedad del conocimiento. En primer lugar, se habla de que los estudiantes deben prepararse para una vida profesional caracterizada por cambios rápidos donde el aprendizaje mediante la experiencia y la interacción con otros es crucial para el éxito económico y la cohesión social. En segundo lugar, se menciona que aquellos que sean de lento aprendizaje (sic) deben recibir un mayor apoyo para poder formar parte de la sociedad y de las actividades económicas. En tercer lugar, se establece el entrenamiento de los adultos como parte de un proceso de aprendizaje a lo largo de la vida como un elemento fundamental en una economía del conocimiento. Como cuarto elemento, se considera que la existencia de una dimensión ética y de contribución a la formación de capital social se vuelve crecientemente importante. Finalmente, el rápido crecimiento en la "producción" de conocimiento en el sector privado puede requerir una nueva división del trabajo y de colaboración entre las escuelas y otros establecimientos de aprendizaje (OCDE, 2000).

Las propuestas educativas de los organismos internacionales como la UNESCO y la OCDE, entre otros, establecen los nuevos paradigmas de la educación en las carreras profesionalizantes. Existe, además, diversa información que trata el tema; pero no se ha encontrado en nuestro país la suficiente referencia al desarrollo de competencias específicas para los administradores en general. De acuerdo con esto, la educación universitaria en el campo de las profesiones no ha permanecido inmóvil ante los retos que ofrecen el contexto globalizador y los grandes avances científicos y tecnológicos. Es un hecho que la nueva sociedad se está desarrollando a una mayor velocidad a la del cambio académico.

\section{Comentarios finales}

Ante las presiones que se generan como consecuencia de la velocidad con que ocurren los cambios - sobre todo aquellos que provienen de las tecnologías de la información y la comunicación y su impacto en el quehacer de las organizaciones, así como los nuevos paradigmas de la economía, originados por la apertura de los mercados y la globalización de éstos- surge la pertinencia de analizar los efectos que se dan en la formación de los administradores, evaluando los requerimientos para el diseño de los planes y programas de estudio. 
Como elementos relevantes de este diseño deben considerarse la incorporación de los métodos didácticos apoyados en las tecnologías de la información y la comunicación, así como la pertinencia de una formación humanista que les dé a los estudiantes una cosmovisión ontológica y les fomente el desarrollo de valores para crearles una mayor sensibilidad sobre la realidad social, económica y política del entorno dentro del cual actúan.

Finalmente, pero no menos importante, existe la necesidad — a la luz de las tendencias educativas basadas en el uso de las tecnologías de la información y la comunicaciónde evaluar el papel que desarrolla el profesor como formador, así como su tradicional misión como modelo y transmisor del conocimiento en el proceso educativo.

\section{Referencias}

Aguilar Camín, Héctor et al. (1992), "I. El estado de la educación”, en Gilberto Guevara Niebla (compilador), La catástrofe silenciosa, Fondo de Cultura Económica, México.

Bennís, W.G. y J. O'Toole (2005), "Cómo las escuelas de negocios perdieron el rumbo", Boston Harvard Business Review, mayo, Boston.

Bowen, J. (1992), Historia de la educación occidental, Tomo III: El occidente moderno, Herder, Barcelona.

Cannac, I. (1984), "La nouvelle enterprise", L'express, 2 noviembre, París, pp. 3743.

Comisión Europea (2006), Alfa-Tuning América Latina, Bélgica [En línea] http:// www.tuning.unideusto.org/tuningal/index.php

Dewey, John, (1995), Democracia y educación, Morata, Madrid.

Friedman, Milton (2001), "Education in the XXI Century", Fortune, septiembre, pp.36-40.

Gómez Oyarzún, Galo (1998), La universidad a través del tiempo, Universidad Iberoamericana, México.

Green, S. (2003), "Executives skills”, Harvard Business Review, agosto, Boston.

Meneses Morales, E. (1979), La Universidad Iberoamericana en el contexto de la educación superior contemporánea, Universidad Iberoamericana, México. 
El reto de la formación profesional de los administradores

MintzBerg, Henry (2004), Managers or MBAs, A hard look at the soft practice of managing and management development, Berrett-Koehler Publishers, San Francisco.

MuÑoz IzQuierdo, Carlos (1992), "II. Un diagnóstico global y VI. El futuro inmediato", en Gilberto Guevara Niebla (compilador), La catástrofe silenciosa, Fondo de Cultura Económica, México.

Organización para la Cooperación y el Desarrollo Económico (ocde) (2000), Knowledge management in the learning society, Centre for Educational Research and Innovation, París.

(2000), Investing in education, oCDe-Unesco, París.

SAVATER, Fernando (1997), El valor De EdUCAR, Ariel, México.

TARr Curtis, W. (1986), "How to humanize MBAs", Fortune, 31 marzo, Nueva York, pp. 11-112.

United Nations Educational, Cientific and Cultural Organization (unesco), (1996), Educación: un tesoro escondido, Comisión Internacional sobre la Educación en el Siglo XXI, París. 